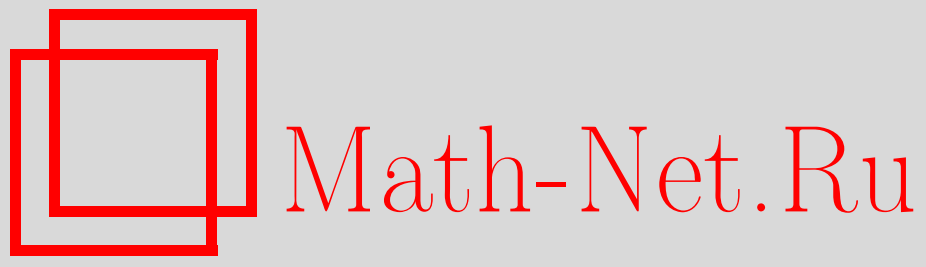

Б. П. Панеях, О разрешимости функциональных уравнений, связанных с динамическими системами с двумя образующими, Функи. анализ и его прил., 2003, том 37, выпуск 1, 55-72

DOI: https://doi.org/10.4213/faa136

Использование Общероссийского математического портала MathNet.Ru подразумевает, что вы прочитали и согласны с пользовательским соглашением

http://www.mathnet.ru/rus/agreement

Параметры загрузки:

IP : 54.81 .137 .203

26 апреля 2023 г., 10:23:10

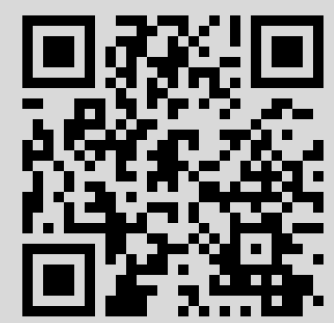




\title{
О разрешимости функциональных уравнений, связанных с динамическими системами с двумя образующими
}

\author{
(C) 2003. Б. П. ПАНЕяХ
}

\section{§1. Введение}

В настоящей работе рассматривается широкий класс вещественных функциональных уравнений вида

$$
F(t)-a_{1}(t) F\left(\delta_{1}(t)\right)-a_{2}(t) F\left(\delta_{2}(t)\right)=h(t), \quad t \in I .
$$

Здесь $I \subset \mathbb{R}$ - отрезок конечной длины, $\delta_{1}$ и $\delta_{2}$ суть непрерывные отображения этого отрезка в себя и $a_{1}, a_{2}, h$ - заданные, а $F-$ искомая вещественные функции на $I$. Это уравнение естественно рассматривать как аналог широко известного когомологического уравнения $F(t)-F(\delta(t))=h(t)$, возникающего в связи с многими вопросами теории динамических систем и эргодической теории. Существенное отличие уравнения (1) состоит в том, что в него входит пара отображений, а возникающая динамика определяется некоммутативной полугруппой с двумя образующими. В связи с этим стоит отметить лемму 3 , дающую описание некоторых регулярных свойств орбит этой полугруппы. Именно эти свойства составляют техническую основу большинства последующих доказательств. Отметим еще, что рассматриваемые уравнения не только вызывают самостоятельный интерес как объект анализа, но оказываются адекватным техническим средством при исследовании ряда новых задач в теории функциональных и интегральных уравнений, а также в теории краевых задач для гиперболических дифференциальных уравнений высокого $(>2)$ порядка. Этот факт находит частичное отражение ниже, в $\$ \S 5-7$.

В заключение я приношу искреннюю благодарность проф. А. М. Вершику за весьма полезное обсуждение динамических аспектов работы и за конструктивную критику ранней версии этой статьи, а также проф. Ю. И. Любичу за многочисленные полезные обсуждения, стимулирующую роль которых трудно переоценить.

\section{§2. Определения и обозначения}

На протяжении всей работы мы используем обозначения

$$
I=\{t \mid-1 \leqslant t \leqslant 1\} \quad \text { и } \quad \stackrel{\circ}{I}=\{t \mid-1<t<1\} .
$$

Относительно параметров, определяющих уравнение (1), предполагается, что

(i) обе функции $\delta_{1}$ и $\delta_{2}$ не убывают на $I$;

(ii) выполняются соотношения $\delta_{2}(t)<t<\delta_{1}(t), t \in \stackrel{\circ}{I}$;

(iii) функции $\delta_{1}$ и $\delta_{2}$ отображают $I$ на отрезки $[0,1]$ и $[-1,0]$ соответственно;

(iv) коэффициенты $a_{1}(t)$ и $a_{2}(t)$ неотрицательны и удовлетворяют условию

$$
0<a_{1}(t)+a_{2}(t) \leqslant 1, \quad t \in I .
$$


Из условий (ii) и (iii) сразу следует, что

$$
\delta_{1}(-1)=\delta_{2}(1)=0, \quad \delta_{1}(1)=1, \quad \delta_{2}(-1)=-1 .
$$

Введем в рассмотрение направляющие множества

$$
\mathscr{T}_{1}=\left\{t \in I \mid a_{2}(t)=0\right\}, \quad \mathscr{T}_{2}=\left\{t \in I \mid a_{1}(t)=0\right\},
$$

и пусть $\mathscr{T}=\mathscr{T}_{1} \cup \mathscr{T}_{2}$. Точки множества $\mathscr{T}$ будем называть $\mathscr{T}$-направляющими. Из условия (iv) следует, что $\mathscr{T}_{1} \cap \mathscr{T}_{2}=\varnothing$.

Заключительное предположение касается взаимных свойств функций $a_{j}$ и $\delta_{k}$ :

(v) $a_{j}(t)=0$ на каждом интервале постоянства функции $\delta_{j}(t), j=1,2$.

В частности, если функция $\delta_{j}(t)$ возрастает, то, помимо неравенств из условия (iv), никакие ограничения на функцию $a_{j}(t)$ не налагаются. Тот факт, что условия (i)-(v) не являются искусственными, подтверждается тем, что уравнения вида (1), возникавшие до сих пор в связи с различными задачами геометрии, теории интегральных и функциональных уравнений, а также в теории краевых задач для гиперболических дифференциальных уравнений, удовлетворяли этим условиям (см., в частности, \$§5-7).

Отображения $\delta_{1}$ и $\delta_{2}$ порождают некоммутативную полугруппу $\Phi_{\delta}$. Элементами этой полугруппы являются всевозможные отображения отрезка $I$ в себя вида $\delta_{J}=\delta_{j_{n}} \circ \ldots \circ \delta_{j_{1}}$, где $J=\left(j_{1}, \ldots, j_{n}\right)$ - мультииндекс, все элементы $j_{k}$ которого равны 1 или 2. Легко проверяется, что в общем случае эта полугруппа не является свободной. Полугруппа $\Phi_{\delta}$ естественно определяет динамическую систему. Мы будем использовать следующую геометрическую терминологию, связанную с $\Phi_{\delta}$ и не вполне совпадающую с традиционной терминологией.

1) Фиксируем произвольное отображение $\delta_{J}$ в $I$ с $J=\left(j_{1}, \ldots, j_{n}\right)$. Упорядоченное множество точек $\mathscr{O}=\left(t_{1}, t_{2}, \ldots, t_{n+1}\right)$ в $I$ называется орбитой (или иногда орбитой точки $t_{1}$ ), если при всех $k, 1 \leqslant k \leqslant n<\infty$, мы имеем

$$
t_{k+1}=\delta_{j_{k}}\left(t_{k}\right) \text {. }
$$

В дальнейшем термин «орбита» используется также для описания бесконечных последовательностей вида $\left(t_{1}, t_{2}, \ldots\right)$, удовлетворяющих условию $(3)$

2) Орбита $\left(t_{1}, \ldots, t_{n+1}\right), n=1,2 \ldots$, называется $\mathscr{T}$-правильной, если в (3)

$$
\delta_{j_{k}}=\delta_{1}, \quad \text { когда } t_{k} \in \mathscr{T}_{1}, \quad \text { и } \quad \delta_{j_{k}}=\delta_{2}, \quad \text { когда } t_{k} \in \mathscr{T}_{2} .
$$

3) Любая орбита, целиком состоящая из $\mathscr{T}$-направляющих точек, называется Т-направленной орбитой.

4) Любая орбита $\mathscr{O}=\left(t_{1}, t_{2}, \ldots, t_{n+1}\right)$, в которой $t_{1}=t_{n+1}$, называется периодической или циклом.

ОПредЕлениЕ. Мы обозначаем через $\mathfrak{N}_{\delta}^{\mathscr{T}}$ множество всех $\mathscr{T}$-направленных T-правильных периодических орбит в $I$.

\section{§3. Разрешимость однородного уравнения (1)}

Основной результат настоящего параграфа составляет теорема 1, описывающая условия и характер разрешимости уравнения (1), в котором $h=0$. Ее доказательство основано на принципе максимума для рассматриваемого класса функциональных уравнений, который представляет и самостоятельный интеpec. В свою очередь, доказательство принципа максимума базируется на изучении некоммутативной динамической системы, порождаемой полугруппой $\Phi_{\delta}$. 
Этот динамический подход позволяет обойти аналитические трудности, нередко возникающие в теории функциональных уравнений, и при этом получить результаты большой степени общности. Его возможности заведомо не исчерпываются решенными в этой статье задачами, и, например, варьирование направляющего множества $\mathscr{T}$ в сочетании с описанием $\mathscr{T}$-правильных аттракторов соответствующей динамической системы может оказаться продуктивным в близких задачах. Обозначим через $\mathscr{T}_{j}^{\prime}, j=1,2$, совокупность предельных точек множества $\mathscr{T}_{j}$, и если оба множества $\mathscr{T}_{1}$ и $\mathscr{T}_{2}$ бесконечны, то положим $\tau_{1}=\min \left\{t \mid t \in \mathscr{T}_{1}^{\prime}\right\}$, $\tau_{2}=\max \left\{t \mid t \in \mathscr{T}_{2}^{\prime}\right\}$. В дальнейшем предполагается, что

$$
\tau_{1}>\tau_{2} .
$$

TEOPEMA $1 . \quad 1^{\circ}$. Пy $\mathrm{cmb}$

$$
a_{1}(t)+a_{2}(t)=1 \quad \text { на } I,
$$

причем

$$
a_{1}(-1) a_{2}(1) \neq 0 .
$$

тогда при выполнении условия

$$
\mathfrak{N}_{\delta}^{\mathscr{T}}=\varnothing
$$

все решения однородного уравнения

$$
F(t)-a_{1}(t) F\left(\delta_{1}(t)\right)-a_{2}(t) F\left(\delta_{2}(t)\right)=0
$$

являются постоянными функцииями.

$2^{\circ}$. Пусть

$$
0<a_{1}(t)+a_{2}(t) \leqslant 1 \quad \text { на } I,
$$

причем множество $\mathscr{B}=\left\{t \mid a_{1}(t)+a_{2}(t)<1\right\}$ содержит некоторую проколотую окрестность ${ }^{1)}$ границь дI. Тогда при выполнении условия (7) уравнение (8) не имеет нетривиальных решений.

Теорема 2 (принцип максимума). $1^{\circ}$. Eсли выполняются условия (5), (6) u (7), то любое решение уравнения (8) принимает максимальное и минимальное значения на границе дI интервала $I$.

$2^{\circ}$. Допустим, что множество $\mathscr{B}$ непусто. Тогда любое решение уравнения (8) принимает положительное максимальное и отрицательное минимальное значения на множестве $I \backslash \mathscr{B}$. При выполнении условия (7) указанные экстремальные значения достигаются на границе дI.

Мы получим этот результат как следствие одного общего утверждения, связанного с аттракторами динамической системы, порожденной полугруппой $\Phi_{\delta}$, и представляющего самостоятельный интерес. Это утверждение никоим образом не связано с уравнением (1). Однако для краткости мы сохраняем все предыдущие обозначения, определения и связывающие их условия за единственным исключением: в следующей ниже лемме 3 в отличие от предыдущего $\mathscr{T}_{1}$ и $\mathscr{T}_{2}$ обозначают произвольные замкнутые множества в $I$ без общих точек, которые содержат все интервалы постоянства функций $\delta_{2}$ и $\delta_{1}$ соответственно. В частности, множество $\mathfrak{N}_{\delta}^{\mathscr{T}}$ состоит в этом случае из периодических $\mathscr{T}$-правильных орбит, целиком расположенных в множестве $\mathscr{T}=\mathscr{T}_{1} \cup \mathscr{T}_{2}$.

1) Если $U-$ окрестность точки $x$, то $U \backslash\{x\}-$ проколотая окрестность этой точки. 
ЛЕмма 3. При выполнении условий (4) и (7) у каждой точки $t_{1} \in I$ существует $\mathscr{T}$-правильная орбита $\mathscr{O}=\left(t_{1}, t_{2}, \ldots\right)$, сходящаяся $\kappa$ одной из точек границь $\partial I$.

ДокАЗАТЕЛЬСтво. Если одно из множеств $\mathscr{T}_{j}$ (скажем, $\mathscr{T}_{2}$ ) пусто, то доказательство очевидно: орбита $\mathscr{O}=\left(t_{1}, \delta_{1}\left(t_{1}\right), \delta_{1}^{2}\left(t_{1}\right), \ldots\right)$ является $\mathscr{T}$-правильной и сходится к точке $t=1$. Последнее вытекает из того, что последовательность $\delta_{1}\left(t_{1}\right), \delta_{1}^{2}\left(t_{1}\right), \ldots$ возрастает, согласно правому неравенству в (ii), и ограничена. Пусть $\lim \delta_{1}^{k}\left(t_{1}\right)=\xi$ при $k \rightarrow \infty$. Тогда $\lim \delta_{1}^{k+1}\left(t_{1}\right)=\delta_{1}(\xi)$, а значит, $\delta_{1}(\xi)=\xi$ и в силу (ii) $\xi=1$.

В последующей части доказательства существенным оказывается

Предложение 4. Если периодическая орбита $\mathscr{C}$ представляет собой часть $\mathscr{T}$-правильной орбиты $\mathscr{O}=\left(t_{1}, t_{2}, \ldots\right)$, то $t_{1} \in \mathscr{C} u t_{1} \neq 0$.

Иными словами, у $\mathscr{T}$-правильных орбит нет точек самопересечения.

ДОКАЗАТЕЛЬСтво ПРЕДЛОЖЕНИЯ 4 . ДоПустим, что $t_{1} \notin \mathscr{C}$, и Пусть $t_{q}, q \geqslant 2$, является первой точкой орбиты $\mathscr{O}$, принадлежащей $\mathscr{C}$. Тогда $t_{q} \neq 0$ и $\mathscr{C}=$ $\left(t_{q}, t_{q+1}, \ldots, t_{q+m}\right)$, где $t_{q+m}=t_{q}$. Очевидно, что $t_{q-1} \neq t_{q+m-1}$, так как $t_{q-1} \notin \mathscr{C}$, а $t_{q+m-1} \in \mathscr{C}$. Допустим, что $t_{q}=\delta_{j_{1}}\left(t_{q-1}\right)$ и $t_{q+m}=\delta_{j_{2}}\left(t_{q+m-1}\right)$. Поскольку $t_{q}=t_{q+m} \neq 0$, из условия (iii) следует, что $j_{1}=j_{2}$. Обозначим общее значение этих чисел через $j$. Очевидно в силу условия (i), что $\delta_{j}(t) \equiv$ const, если $t_{q-1} \leqslant t \leqslant t_{q+m-1}$. Это означает на основании определения множеств $\mathscr{T}_{1}$ и $\mathscr{T}_{2}$, что $t_{q-1} \in \mathscr{T}_{j^{\prime}}$ с $j^{\prime} \neq j$, в противоречие с тем, что орбита $\left(t_{q-1}, t_{q}\right)$ является $\mathscr{T}$-правильной. Предложение 4 тем самым доказано.

Продолжая доказательство леммы 3 , отметим, что, как следует из неравенства (4), при достаточно малом значении $\varepsilon>0$ существуют проколотые окрестности $U_{1}^{\varepsilon}$ и $U_{2}^{\varepsilon}$ точек $t=-1$ и $t=1$ соответственно, такие, что $U_{1}^{\varepsilon} \cap \mathscr{T}_{1}=\varnothing$ и $U_{2}^{\varepsilon} \cap \mathscr{T}_{2}=\varnothing$. Очевидно, что если $t \in U_{1}^{\varepsilon}$ или $t \in U_{2}^{\varepsilon}$, то орбита $\mathscr{O}_{-}=$ $\left\{t, \delta_{2}(t), \delta_{2}^{2}(t), \ldots\right\}$ или $\mathscr{O}_{+}=\left\{t, \delta_{1}(t), \delta_{1}^{2}(t), \ldots\right\}$ соответственно оказывается $\mathscr{T}$-правильной и, как доказано выше, сходится соответственно к точке $t=-1$ или $t=1$.

Легко видеть, что для указанного числа $\varepsilon$ существует такое число $\nu$, что для всех точек $t \in I$ справедливы включения $\delta_{2}^{\nu}(t) \in U_{1}^{\varepsilon}$ и $\delta_{1}^{\nu}(t) \in U_{2}^{\varepsilon}$. В самом деле, в случае отображения $\delta_{1}$, например, неравенство $\delta_{1}^{\nu}(-1)>1-\varepsilon$ выполняется при некотором $\nu$ на основании условия (ii). Требуемое включение теперь немедленно следует из условия монотонности (i).

Выберем произвольную точку $\tau_{0}$ между $\tau_{1}$ и $\tau_{2}$ (что возможно в силу условия (4)) и рассмотрим открытое покрытие множества $\mathscr{T}^{\prime}=\mathscr{T}_{1}^{\prime} \cup \mathscr{T}_{2}^{\prime}$ непересекающимися окрестностями $V_{1}$ и $V_{2}$, такими, что

1) $V_{1} \supset \mathscr{T}_{1}^{\prime}, V_{2} \supset \mathscr{T}_{2}^{\prime}, V_{1} \cap \mathscr{T}_{2}=\varnothing, V_{2} \cap \mathscr{T}_{1}=\varnothing$;

2) множество $V_{1}$ лежит справа, а множество $V_{2}-$ слева от точки $\tau_{0}$.

Существование такого покрытия не вызывает сомнений. Положим $V=V_{1} \cup V_{2}$. Важно отметить, что множество $W=\mathscr{T} \backslash V$ является конечным. Обозначим через W число точек в $W$. Условимся любую $\mathscr{T}$-правильную орбиту $\mathscr{O}=\left(t_{1}, t_{2}, \ldots\right)$ называть направленной, если в равенстве (3)

$$
\delta_{j_{k}}= \begin{cases}\delta_{1}, & \text { когда } t_{k} \notin \mathscr{T}_{2} \text { и } t_{k} \geqslant \tau_{0}, \\ \delta_{2}, & \text { когда } t_{k} \notin \mathscr{T}_{1} \text { и } t_{k}<\tau_{0} .\end{cases}
$$


Очевидно, что каждая направленная орбита определяется однозначно своей первой точкой. Условимся называть точкой поворота орбиты $\mathscr{O}=\left(t_{1}, t_{2}, \ldots\right)$ такую точку $t_{n}$, для которой $t_{n}=\delta_{j}\left(t_{n-1}\right)$ и $t_{n+1}=\delta_{j^{\prime}}\left(t_{n}\right)$ при $j \neq j^{\prime}$. Непосредственно проверяется, что в направленной орбите $\mathscr{O}=\left(t_{1}, t_{2}, \ldots\right)$ каждой точке поворота $t_{N} \in V$ предшествует точка $t_{N-1} \in W$ и то же самое верно для каждой точки поворота $t_{N} \in I \backslash \mathscr{T}$. Поскольку $I=V \cup(I \backslash \mathscr{T}) \cup W$, сказанное выше означает, что число точек поворота в орбите $\mathscr{O}$ не превосходит W, если эта орбита не содержит периодических суборбит. Но тогда становится очевидным, что последняя точка в направленной орбите $\mathscr{O}_{1}=\left(t_{1}, t_{2}, \ldots, t_{N}\right)$, где $N=\mathbf{W} \nu+\mathbf{W}+\nu$, лежит вне интервала $(-1+\varepsilon, 1-\varepsilon)$. Поэтому одна из сшитых орбит $\mathscr{O}=\left(t_{1}, \ldots, t_{N}, \delta_{j}\left(t_{N}\right), \delta_{j}^{2}\left(t_{N}\right), \ldots\right)$ с $j=1$ или $j=2$ обладает свойствами, постулированными в лемме 3 .

Допустим теперь, что направленная орбита $\mathscr{O}$ точки $t_{1}$ включает некоторый цзикл $\mathscr{C}$. На основании предложения 4 этот цикл содержит точку $t_{1}$ и, следовательно, имеет вид $\mathscr{C}=\left(t_{1}, t_{2}, \ldots, t_{m}\right)$, где $t_{m}=t_{1}$. Поскольку $\mathfrak{N}_{\delta}^{\mathscr{T}}=\varnothing$, орбита $\mathscr{C}$ не является $\mathscr{T}$-направленной. Поэтому одна из ее точек, $t_{q}, 1 \leqslant q \leqslant m-1$, не принадлежит множеству $\mathscr{T}$. Допустим, что $t_{q+1}=\delta_{j}\left(t_{q}\right)$. Введем в рассмотрение новую точку $\hat{t}_{q+1}=\delta_{j^{\prime}}\left(t_{q}\right)$ с $j^{\prime} \neq j$ и определим направленную орбиту $\mathscr{O}_{1}=\left(\hat{t}_{q+1}, \hat{t}_{q+2}, \ldots\right)$. Будучи $\mathscr{T}$-правильной, эта орбита не имеет общих точек с циклом $\mathscr{C}$. В самом деле, если $\hat{t}_{p}$ является первой такой точкой и $\hat{t}_{p}=t_{r}$, $1 \leqslant r \leqslant m-1$, то, как мы видели при доказательстве предложения $4, \hat{t}_{p-1}=t_{r-1}$. Это, однако, невозможно, так как $t_{r-1} \in \mathscr{C}$, в то время как $\hat{t}_{p-1} \notin \mathscr{C}$. В силу того же предложения $4 \mathscr{T}$-правильная орбита $\left(t_{q}, \hat{t}_{q+1}, \hat{t}_{q+2}, \ldots\right)$ не содержит циклических суборбит. Это позволяет заключить, как и выше, что сшитая орбита

$$
\mathscr{O}_{j}=\left(t_{1}, t_{2}, \ldots, t_{q}, \hat{t}_{q+1}, \ldots, \hat{t}_{q+N}, \delta_{j}\left(\hat{t}_{q+N}\right), \delta_{j}^{2}\left(\hat{t}_{q+N}\right), \ldots\right),
$$

где $N=\mathbf{W} \nu+\mathbf{W}+\nu, j=1$, если $\hat{t}_{q+N}>\tau_{0}$, и $j=2$, если $\hat{t}_{q+N} \leqslant \tau_{0}$, отвечает всем требованиям, сформулированным в лемме 3. Это завершает доказательство леммы.

ДОКАЗАТЕЛЬСтво ТЕОРЕмы 2. $1^{\circ}$. Пусть $M=\max _{I} F$ и $\mathscr{M}=\{t \in I \mid$ $F(t)=M\}$. Если $F(\hat{t})=M$, то $F\left(\delta_{1}(\hat{t})\right)=M-\varepsilon_{1}, F\left(\delta_{2}(\hat{t})\right)=M-\varepsilon_{2}$ для некоторых неотрицательных $\varepsilon_{1}$ и $\varepsilon_{2}$. Заменяя в уравнениии $(8) t$ на $\hat{t}$, мы получим равенство $a_{1}(\hat{t}) \varepsilon_{1}+a_{2}(\hat{t}) \varepsilon_{2}=0$. Отсюда следует, что если $\hat{t} \in \mathscr{M} \backslash \mathscr{T}$, то $\varepsilon_{1}=\varepsilon_{2}=0$, а потому $\delta_{1}(\hat{t}) \in \mathscr{M}$ и $\delta_{2}(\hat{t}) \in \mathscr{M}$. Если же $\hat{t} \in \mathscr{M} \cap \mathscr{T}_{1}$, то $\varepsilon_{1}=0$ и, значит, $\delta_{1}(\hat{t}) \in \mathscr{M}$. Точно так же, если $\hat{t} \in \mathscr{M} \cap \mathscr{T}_{2}$, то $\delta_{2}(\hat{t}) \in \mathscr{M}$. Сопоставляя эти наблюдения с определением $\mathscr{T}$-правильной орбиты (см. §2), мы заключаем, что вместе с каждой точкой $t_{1} \in \mathscr{M}$ тому же множеству $\mathscr{M}$ принадлежит следующая точка $t_{2}$ любой $\mathscr{T}$-правильной орбиты $\left(t_{1}, t_{2}\right)$. Поскольку $\delta_{1}$ и $\delta_{2}$ отображают отрезок $I$ в себя, это рассуждение можно применить к точке $t_{2}$ и получить одну или две точки $t_{3}$ вида $t_{3}=\delta_{j_{2}} \circ \delta_{j_{1}}\left(t_{1}\right)$, лежащие в множестве $\mathscr{M}$. При этом орбита $\mathscr{O}=\left(t_{1}, t_{2}, t_{3}\right)$ оказывается $\mathscr{T}$-правильной. Поступая так и далее, мы приходим к заключению, что вместе с каждой точкой $t_{1} \in \mathscr{M}$ любая ее $\mathscr{T}$-правильная орбита лежит в $\mathscr{M}$. Согласно лемме 3 , хотя бы одна из этих орбит сходится либо к точке $t=-1$, либо к точке $t=1$. Поскольку функция $F$ непрерывна, доказано, что одно из чисел $F(1)$ или $F(-1)$ равно $M$. Заметим теперь, что сказанное выше относительно распространения максимального значения решения вдоль $\mathscr{T}$-правильных орбит остается справедливым для 
минимального значения $m$ того же решения $F$. В результате мы устанавливаем, что одно из чисел $F(1)$ или $F(-1)$ равно $m$, чем и завершается доказательство п. $1^{\circ}$ теоремы.

$2^{\circ}$. Пусть $F-$ произвольное решение уравнения $(8)$, и пусть $M=F(\hat{t})>0$. Если $\hat{t} \in \mathscr{B}$, то, как следует из $(8), M \leqslant a_{1}(\hat{t}) M+a_{2}(\hat{t}) M<M$, что невозможно. Таким образом, доказано, что положительный максимум решения $F$ в рассматриваемых условиях достигается только на множестве $I \backslash \mathscr{B}$. Точно так же устанавливается, что отрицательный минимум решения $F$ достигается в точках множества $I \backslash \mathscr{B}$. Заключительное утверждение доказывается дословным повторением доказательства утверждения $1^{\circ}$. Теорема 2 доказана полностью.

ДокАЗАТЕЛЬСтво теОРемы 1. Заметим, что любое решение уравнения (8) удовлетворяет «граничным» условиям

$$
F(-1)=F(1)=F(0) .
$$

Чтобы в этом убедится, достаточно подставить в уравнение (8) последовательно $t=-1$ и $t=1$ и воспользоваться равенствами (2) и (5), а также условием (6). Для доказательства утверждения $1^{\circ}$ заметим, что, согласно принципу максимума, каждое из чисел $M=\max F, m=\min F$ совпадает с одним из чисел $F(-1)$, $F(1)$. В сочетании с (10) это дает $M=m$, а значит $F \equiv$ const.

В случае $2^{\circ}$, согласно п. $2^{\circ}$ теоремы 2 , решение $F$ уравнения $(8)$ не может принимать ни положительного максимального, ни отрицательного минимального значений. Но это возможно только в случае, если $F \equiv 0$. Теорема 1 доказана.

Следующее утверждение можно трактовать как некое осцилляционное свойство решений уравнения (8).

Теорема 5. Если множество ஜ̈ непусто, то при выполнении условий (6) $u$ (7) любое решение уравнения (8) не меняет знак на I. При этом неотрицательное решение достигает минимума только в точках множества Я̈ и то же верно относительно максимума неположительного решения.

ДоказАтельство. Допустим, что решение $F$ уравнения (8) принимает значения разных знаков. Тогда

$$
M=\max F>0 \quad \text { и } \quad m=\min F<0 .
$$

В силу теоремы 2 эти же значения принимаются функцией $F$ на границе $\partial I$, и, кроме того, $\partial I \subset I \backslash \mathscr{B}$. Это, однако, невозможно ввиду равенства (10).

Предположим, что $F \geqslant 0$ на $I$, и пусть $F(\breve{t})=m$. Если $\breve{t} \in I \backslash \mathscr{B}$, то, повторяя доказательство теоремы 2 , мы получаем, что одно из чисел $F(-1)$ или $F(1)$ равно $m$. В силу той же теоремы число $M$ также совпадает либо с $F(-1)$, либо с $F(1)$. Но тогда в силу (10) $M=m$ и, значит, $F \equiv$ const, а это невозможно, если $\mathscr{B} \neq \varnothing$. Таким образом, $\breve{t} \in \mathscr{B}$, что и завершает доказательство теоремы.

\section{§4. Разрешимость неоднородного уравнения (1)}

В этом параграфе рассматриваются вопросы существования решений неоднородного уравнения (1) при выполнении условия (9) и при его нарушении.

ТЕОрема 6. Если множество $\mathscr{B}$ содержит непустую проколотую окрестность границьь ӘI, то при выполнении условия (7) уравнение (1) имеет единственное решение $F \in C(I)$ для любой функциии $h \in C(I)$. 
ДокАЗАТЕЛЬСтво. Введем в пространстве $C(I)$ линейный оператор $L: F(t) \mapsto$ $a_{1}(t) F\left(\delta_{1}(t)\right)+a_{2}(t) F\left(\delta_{2}(t)\right)$, и пусть $E$ обозначает тождественный оператор. Хорошо известно, что если норма $\left\|L^{m}\right\|$ оператора $L^{m}$ меньше 1 при некотором целом $m>0$, оператор $E-L$ обратим. Поэтому теорема 6 будет доказана, если такое число $m$ будет предъявлено в случае рассматриваемого оператора $L$. Заметим, прежде всего, что для любого целого числа $N>0$ функция $L^{N} F$ может быть представлена в виде

$$
L^{N} F(t)=\sum_{j_{1}, \ldots, j_{N}=1}^{2} a_{j_{1}}(t) a_{j_{2}}\left(\delta_{j_{1}}(t)\right) \cdots a_{j_{N}}\left(\delta_{j_{N-1}} \circ \cdots \circ \delta_{j_{1}}(t)\right) F\left(\delta_{J}(t)\right),
$$

где $J=\left(j_{1}, \ldots, j_{N}\right)$. В самом деле, при $N=1$ это очевидно. Чтобы воспользоваться индукцией по $N$, допустим, что представление (11) верно для некоторого $N$, и докажем его справедливость для $N+1$. Из определения оператора $L$ и формулы (11) следует, что

$$
\begin{aligned}
\left(L^{N+1} F\right)(t) & =\left(L \circ L^{N}\right) F(t)=\sum_{j_{0}=1}^{2} a_{j_{0}}(t)\left(L^{N} F\right)\left(\delta_{j_{0}}(t)\right) \\
& =\sum_{j_{0}, j_{1}, \ldots, j_{N}=1}^{2} a_{j_{0}}(t) a_{j_{1}}\left(\delta_{j_{0}}(t)\right) \cdots a_{j_{N}}\left(\delta_{j_{N-1}} \circ \cdots \circ \delta_{j_{0}}(t)\right) F\left(\delta_{J}(t)\right),
\end{aligned}
$$

где $J=\left(j_{0}, j_{1}, \ldots, j_{N}\right)$. Остается заметить, что, заменяя $j_{k}$ на $j_{k+1}, k=0, \ldots, N$, в этом равенстве, мы получаем формулу (11) с $N+1$ вместо $N$.

Как следует из (11), для произвольной функции $F$ с $\|F\|=1$ в каждой точке $t \in I$ справедливо неравенство

$$
\left|L^{N} F(t)\right| \leqslant \sum_{j_{1}, \ldots, j_{N}=1}^{2} a_{j_{1}}(t) a_{j_{2}}\left(\delta_{j_{1}}(t)\right) \cdots a_{j_{N}}\left(\delta_{j_{N-1}} \circ \cdots \circ \delta_{j_{1}}(t)\right) .
$$

Докажем, что для любого фиксированного $t$ можно найти целое число $N$ и константу $\gamma<1$, такие, что для тех же функций $F$ выполняется неравенство

$$
\left|L^{N} F(t)\right|<\gamma .
$$

Если $t \in \mathscr{B}$, то справедливость этого утверждения при $N=1$ следует из определения множества $\mathscr{B}$. Если $t \notin \mathscr{B}$, то рассмотрим произвольную $\mathscr{T}$-правильную орбиту $\mathscr{O}=\left(t_{1}, t_{2}, \ldots\right)$ с $t_{1}=t$, сходящуюся к одной из граничных точек интервала $I$. Существование такой орбиты доказано в лемме 3 . Согласно условию теоремы, при достаточно большом значении $N$ точка $t_{N}=\delta_{j_{N-1}} \circ \cdots \circ \delta_{j_{1}}(t)$ оказывается внутри множества $\mathscr{B}$, так что

$$
a_{1}\left(t_{N}\right)+a_{2}\left(t_{N}\right)<1 \quad \text { и } \quad a_{j_{1}}(t) a_{j_{2}}\left(\delta_{j_{1}}(t)\right) \cdots a_{j_{N-1}}\left(\delta_{j_{N-2}} \circ \cdots \circ \delta_{j_{1}}(t)\right) \neq 0 .
$$

Последнее неравенство следует из определения $\mathscr{T}$-правильной орбиты. Покажем, что при таком выборе $N$ неравенство (13) справедливо. Введем обозначения $a_{j_{k}}\left(\delta_{j_{k-1}} \circ \cdots \circ \delta_{j_{1}}(t)\right)=a_{j_{1} \ldots j_{k}}, k=1, \ldots, N$, где $\delta_{j_{0}}(t)=t_{1}$. Рассмотрим правую часть в неравенстве (12) как $N$-линейную форму

$$
Q_{N}=\sum_{j_{1}, \ldots, j_{N}=1}^{2} a_{j_{1}} a_{j_{1} j_{2}} \cdots a_{j_{1} \ldots j_{N}}
$$


неотрицательных переменных $a_{j_{1} \ldots j_{k}}, k=1, \ldots, N$, удовлетворяющих условию $\sum_{j_{k}=1}^{2} a_{j_{1} \ldots j_{k}} \leqslant 1$ при всех $k$. Очевидно, что $0<Q_{N} \leqslant 1$ при любых $N$. Легко проверяется, что если хотя бы для одного мультииндекса $J=\left(j_{1}, \ldots, j_{N-1}\right)$ выполняются условия

$$
a_{j_{1}} a_{j_{1} j_{2}} \cdots a_{j_{1} \ldots j_{N-1}} \neq 0 \quad \text { и } \quad \sum_{j_{N}=1}^{2} a_{j_{1} \ldots j_{N}}<1,
$$

то $Q_{N}<1$. При $N=2$ утверждение очевидно. В предположении, что оно верно для $N$, докажем его справедливость для $N+1$. С этой целью запишем форму $Q_{N+1}$ в виде $Q_{N+1}=a_{1} Q_{N}^{(1)}+a_{2} Q_{N}^{(2)}$, где $Q_{N}^{(1)}$ и $Q_{N}^{(2)}-$ соответствующие $N$-линейные формы переменных $a_{1 j_{2} \ldots j_{k}}$ и $a_{2 j_{2} \ldots j_{k}}, k=2, \ldots, N+1$. Условия (15) применительно к форме $Q_{N+1}$ означают, как легко видеть, что хотя бы одна из форм $Q_{N}^{(j)}$ удовлетворяет тем же условиям и при этом $a_{j} \neq 0$. В силу предположения индукции $Q_{N}^{(j)}<1$, а так как $a_{1}+a_{2} \leqslant 1$ и $Q_{N}^{\left(j^{\prime}\right)} \leqslant 1$ при $j^{\prime} \neq j$, то неравенство $Q_{N+1}<1$ становится очевидным. Для завершения доказательства неравенства (13) остается заметить, что условие (14) есть не что иное, как неравенства (15).

Заметим теперь, что в силу непрерывности всех рассматриваемых функций неравенство (13) выполняется с тем же числом $N$, хотя и с большей константой $\gamma<1$, во всех точках некоторой окрестности $U$ рассматриваемой нами точки $t$. Совокупность таких окрестностей образует открытое покрытие замкнутого множества $I \backslash \mathscr{B}$. Пусть $\left\{U_{j}\right\}_{j=1}^{k}-$ конечная подсистема этих окрестностей и $N_{j}, \gamma_{j}$ - соответствующие константы. Полагая $m=\max N_{j}$ и $\gamma=\max \gamma_{j}$, мы приходим к требуемому неравенству $\left\|L^{m}\right\|<1$, что и завершает доказательство теоремы 6 .

Широкий класс уравнений (1), обладающих свойствами разрешимости, постулированными в теореме 6 , описан в следующем утверждении.

СледСтвиЕ 7. Пусть $\left(0, \varepsilon_{1}, \varepsilon_{2}, \ldots\right)$ - произвольная Я-правильная орбита точки $\varepsilon_{0}=0$. Результат теоремь 6 остается верным, если в формулировке этой теоремь границу ӘІ заменить произвольной точкой $\varepsilon_{k}, k \geqslant 0$.

Несложное доказательство этого факта основано на лемме 3 и теореме 6, и мы не будем его здесь приводить.

В завершение этого параграфа приведем еще один результат, касающийся разрешимости уравнения (1) и в известном смысле дополняющий теорему 5.

ТЕОРЕмА 8. Пусть $a_{1}(t)+a_{2}(t)=1$ всюду на I и выполняются условия (6) и (7). Тогда уравнение (1) не имеет решений, если функиия $h$ не меняет знак на I и отлична от нуля в сколь угодно малой проколотой окрестности границьь $\partial I$.

ДокАЗАТЕЛьство. Пусть $F$ - решение уравнения (1) при заданной функции $h$ и $F(\hat{t})=M, F(\breve{t})=m-$ максимальное и минимальное значения функции $F$ соответственно. Подставляя в (1) последовательно $t=\hat{t}$ и $t=\breve{t}$, мы получим, как при доказательстве теоремы 2 , неравенства $h(\hat{t}) \geqslant 0$ и $h(\breve{t}) \leqslant 0$. Таким образом, максимальное и минимальное значения функции $h$ не могут быть одного знака. Допустим, что $h \geqslant 0$ на $I$ и $h>0$ в некоторой проколотой окрестности $U$ границы $\partial I$. Тогда $\min h=0$ и, в частности, $h(\breve{t})=0$. Как следует из доказательства 
теоремы 2 , минимум $m$ решения $F$ распространяется вдоль $\mathscr{T - п р а в и л ь н ы х ~ о р - ~}$ бит точки $\breve{t}$. Следовательно, на каждой из таких орбит функция $h$ обращается в нуль. В силу леммы $3 h=0$ в точках, произвольно близких к границе, что противоречит выбору функции $h$. Это завершает доказательство теоремы 8.

ЗАмЕчАниЕ. Сравнивая результаты теорем 5 и 8, мы видим, что в то время как однородное уравнение (1) не имеет осциллирующих (т.е. принимающих разные знаки) решений $F$, неоднородное уравнение (1) может быть разрешимо только при осциллирующих правых частях $h$.

\section{§5. К теории разрешимости функционального уравнения типа Коши}

Одно из первых функциональных уравнений было рассмотрено Коши. Он доказал, что если непрерывная (в одной точке) функция $F(z),|z| \leqslant 1$, удовлетворяет условию

$$
F(x+y)=F(x)+F(y)
$$

во всех точках $(x, y)$ квадрата $K=\{(x, y)|| x \pm y \mid \leqslant 1\}$, то $F(z)=\lambda z$.

В настоящем параграфе в качестве одного из приложений результатов $\$ \$ 3-4$ мы рассмотрим проблему разрешимости фунцционального уравнения

$$
F\left(\left(\beta_{1}(t)+\beta_{2}(t)\right)-F\left(\beta_{1}(t)\right)-F\left(\beta_{2}(t)\right)=h(t),\right.
$$

где, как и в $\$ 2, \beta_{1}$ и $\beta_{2}$ - заданные непрерывные отображения отрезка $I$ в себя, а $h$ и $F-$ соответственно заданная и неизвестная вещественные функции на $I$. Помимо того, что такие уравнения интересны сами по себе, они возникают как необходимый технический аппарат при решении некоторых интегральных уравнений (см. §6), а также при изучении краевых задач для гиперболических уравнений высокого порядка в ограниченной области (см. §7). Отметим, что неоднородные уравнения (17) рассматриваются в настоящей работе впервые. Если интерпретировать равенства

$$
x=\beta_{1}(t), \quad y=\beta_{2}(t), \quad t \in I,
$$

как параметрическое представление неособой кривой $\Gamma$ на плоскости $\mathbb{R}^{2}$, то по ассоциации с уравнением (16) будет естественно назвать (17) функциональным уравнением типа Коши (на кривой Г). Такой подход к уравнению (17) позволит нам в дальнейшем (см. следствие 10) трактовать основной результат этого параграфа как неожиданное усиление теоремы Коши, касающейся уравнения (16).

Переходим к точной постановке задачи. Рассмотрим линейный оператор $B$ в пространстве $C(I)$ вида

$$
B: F(t) \mapsto F\left(\left(\beta_{1}+\beta_{2}\right)(t)\right)-F\left(\beta_{1}(t)\right)-F\left(\beta_{2}(t)\right),
$$

где $\beta_{1}$ и $\beta_{2}$ суть описанные выше функции на $I$, удовлетворяющие условиям (i)-(iii) (см. $\S 2)$, а также неравенствам $\left.{ }^{1}\right)$

$$
\left(\beta_{1}^{\prime}+\beta_{2}^{\prime}\right)(t)>0, \quad t \in I, \quad \beta_{1}^{\prime} \beta_{2}^{\prime}>0 \quad \text { на } \partial I .
$$

Отсюда вытекает, что отображение $\beta=\beta_{1}+\beta_{2}$ представляет собой диффеоморфизм в $I$, сохраняющий границу $\partial I$. Как следствие этого отображения

$$
\delta_{1}=\beta_{1} \circ \beta^{-1} \quad \text { и } \quad \delta_{2}=\beta_{2} \circ \beta^{-1}
$$

1) При других ограничениях на функции $\beta_{1}$ и $\beta_{2}$ уравнение (17) изучается также в работе [4]. 
отрезка $I$ в себя обладают всеми свойствами одноименных отображений $\delta_{1}$ и $\delta_{2}$, введенных в 2 , и, кроме того,

$$
\delta_{1}^{\prime}+\delta_{2}^{\prime}=1 .
$$

Множества критических точек отображений $\delta_{j}, j=1,2$, мы обозначаем через $\mathscr{T}_{j}$, называем направляющими и полагаем $\mathscr{T}=\mathscr{T}_{1} \cup \mathscr{T}_{2}$. Очевидно, что функции $\delta_{1}^{\prime}(t)$ и $\delta_{2}^{\prime}(t)$ обладают всеми свойствами функций $a_{1}(t)$ и $a_{2}(t)$ (см. $\left.\S 2\right)$, включая свойство (v). Как и в $§ 2$, при наличии двух отображений $\delta_{1}$ и $\delta_{2}$ отрезка $I$ в себя и двух множеств $\mathscr{T}_{1}$ и $\mathscr{T}_{2}$ в $I$ можно ввести полугруппу $\Phi_{\delta}$ и все геометрические определения, связанные с $\Phi_{\delta}$ и множеством $\mathscr{T}$. В частности $\mathfrak{N}_{\delta}^{\mathscr{T}}$ служит обозначением множества всех периодических $\mathscr{T}$-правильных орбит, целиком расположенных в множестве $\mathscr{T}$.

Очевидно, что ядро оператора $B$ включает все линейные функции, а так как коядро этого оператора также непусто (см. (20)), то для получения наилучших результатов о разрешимости уравнения (17) желательно заранее выяснить необходимые условия такой разрешимости. Подставим в (17) последовательно значения $t=-1$ и $t=1$. Используя равенства (2) (которые верны и для функций $\beta_{j}$ ), мы получаем, что для произвольной функции $F \in C(I)$

$$
B F(-1)=B F(1)=-F(0) .
$$

Это означает, что область значений оператора $B$ состоит только из функций $h$, принимающих равные значения в точках границы $\partial I$. Кроме того, неизвестная функция $F$ должна быть связана с заданной функцией $h$ условием

$$
F(0)=-h(-1)=-h(1) .
$$

Учитывая, что решение $F(z)=\lambda z$ уравнения (17), в котором $h=0$, однозначно определяется заданием значения $F(1)$, мы приходим к следующей естественной задаче: для заданных чисел $\lambda, \mu \in \mathbb{R}$ и произвольной функции $h \in C^{2}(I)$, удовлетворяющей условию

$$
h(1)=h(-1)=-\mu,
$$

найти такую функцию $F(t) \in C^{2}(I)$, для которой

$$
B F=h \text { на } I, \quad F(0)=\mu, \quad F(1)=\lambda .
$$

Главный результат настоящего параграфа составляет следующая

ТЕоремА 9. Если $\mathfrak{N}_{\delta}^{\mathscr{T}}=\varnothing$, то при выполнении условия (4), каковы бы ни были константы $\lambda, \mu$ и функция $h \in C^{2}(I)$, удовлетворяющие условию (21), существует единственное решение $F \in C^{2}(I)$ задачи (22). Обратный оператор $h \mapsto F$ является непрерывным оператором из $C^{2}(I)$ в $C^{2}(I)$.

ЗАмЕчАниЕ. Утверждение теоремы остается верным, если в задаче (22) условие $F(1)=\lambda$ заменить условием $F^{\prime}(0)=\lambda$. Микроскопические изменения в доказательстве теоремы, к которым такая замена приводит, вносятся без труда.

ДокАЗАТЕЛЬСтво. Заменяя в задаче (22) функцию $F$ на $F+t(\lambda-\mu)+\mu$, мы приходим к задаче

$$
B F=h_{\mu} \text { на } I, \quad F(0)=0, \quad F(1)=0,
$$


где $h_{\mu}=h+\mu$. Замена переменной $t \rightarrow \beta^{-1}(t)$ в уравнении, входящем в $(23)$, приводит задачу (23) к виду

$$
\widehat{B} F(t):=F(t)-F\left(\delta_{1}(t)\right)-F\left(\delta_{2}(t)\right)=\hat{h}_{\mu}(t), \quad F(0)=0, \quad F(1)=0,
$$

который и является отправной точкой при изучении задачи $(22)$. Как следует из равенств $(20)$ и $(21)$, обе функции $\widehat{B} F$ и $\hat{h}_{\mu}$ равны нулю в точках $t=-1$ и $t=1$. Поэтому, дифференцируя дважды уравнение $\widehat{B} F(t)=\hat{h}_{\mu}(t)$, мы приходим к новым задачам, равносильным задаче (24), а именно

$$
\begin{aligned}
B_{1} F^{\prime}(t) & :=F^{\prime}(t)-\delta_{1}^{\prime}(t) F^{\prime}\left(\delta_{1}(t)\right)-\delta_{2}^{\prime}(t) F^{\prime}\left(\delta_{2}(t)\right)=\hat{h}_{\mu}^{\prime}(t), \\
B_{2} F^{\prime \prime}(t) & :=F^{\prime \prime}(t)-\delta_{1}^{\prime 2}(t) F^{\prime \prime}\left(\delta_{1}(t)\right)-\delta_{2}^{\prime 2}(t) F^{\prime \prime}\left(\delta_{2}(t)\right)-K F^{\prime \prime}(t)=\hat{h}_{\mu}^{\prime \prime}(t),
\end{aligned}
$$

где для краткости опущены условия $F(0)=0, F(1)=0$, а символ $K$ служит обозначением линейного оператора в $C(I)$ вида

$$
K: H \mapsto \delta_{1}^{\prime \prime}(t) \int_{\xi}^{\delta_{1}(t)} H(s) d s+\delta_{2}^{\prime \prime}(t) \int_{\xi}^{\delta_{2}(t)} H(s) d s .
$$

Здесь $\xi$ обозначает любую точку интервала $(0,1)$, в которой $F^{\prime}(\xi)=0$. Существование такой точки $\xi$ обусловлено граничными условиями.

В силу эквивалентности трех указанных задач теорема будет доказана, если мы установим инъективность оператора $B_{1}$, а также тот факт, что индекс оператора $B_{2}$ в пространстве $C(I)$ равен нулю.

Для доказательства инъективности оператора $B_{1}$ на пространстве функций $F$, равных нулю в точках $t=0$ и $t=1$, положим $\hat{h}_{\mu}^{\prime}=0$ в $(25)$. В силу равенства (19) полученное уравнение есть не что иное, как однородное уравнение (1), в котором коэффициенты $a_{j}$ совпадают с $\delta_{j}^{\prime}, j=1,2$, и потому оно удовлетворяет всем условиям теоремы 1 . На основании п. $1^{\circ}$ этой теоремы мы заключаем, что $F^{\prime}=$ const, и так как функция $F$ равна нулю в двух точках, ее производная $F^{\prime}$ равна нулю тождественно.

Переходя к оператору $B_{2}$, представим его в форме $B_{2}=E-L-K$, где $L$ обозначает линейный оператор в пространстве $C(I)$ вида

$$
L: H \mapsto \delta_{1}^{\prime 2} H \circ \delta_{1}+\delta_{2}^{\prime 2} H \circ \delta_{2} .
$$

Как следует из (19), $\delta_{1}^{\prime 2}+\delta_{2}^{\prime 2}<1$ всюду на $I$, кроме точек множества $\mathscr{T}$. Поэтому уравнение $G-L G=h$ представляет собой уравнение (1), в котором коэффициенты $a_{j}$ совпадают с $\delta_{j}^{\prime 2}, j=1,2$, а потому удовлетворяют условию $(9)$. Роль множества $\mathscr{B}$ в этом случае выполняет множество $I \backslash \mathscr{T}$, и, как следует из (18), условие $\partial I \subset \mathscr{B}$ также выполняется для указанных $a_{j}$. Таким образом к рассматриваемому уравнению оказывается применимой теорема 6 , и мы устанавливаем, что оператор $E-L$ обратим (хотя $\|L\|=1$ ). Таким образом, оператор $B_{2}$ оказывается суммой обратимого и компактного операторов в пространстве $C(I)$ и, как следует из теоремы Ф. Рисса и Шаудера, ind $B_{2}=0$. Поскольку $\operatorname{dim} \operatorname{ker} B_{2}=\operatorname{dim} \operatorname{ker} B_{1}=0$, отсюда следует однозначная разрешимость задачи (24). Остается проверить непрерывность обратного оператора в задаче $(22)$. Поскольку оператор $B: C^{2}(I) \rightarrow C^{2}(I)$ непрерывен, отсюда следует на основании теоремы Банаха о замкнутом графике, что оператор $B^{-1}$ непрерывен в $C^{2}(I)$, и это завершает доказательство теоремы. 
СлЕДСтвиЕ 10. В плоскости $\mathbb{R}^{2}$ переменных $x$, у рассмотрим произвольную неубывающую кривую $\Gamma \subset C^{2}$, соединяюшую точки $(0,-1)$ и $(1,0)$ и допускающую параметрическое представление вида $x=\beta_{1}(t), y=\beta_{2}(t),-1 \leqslant t \leqslant 1$, в котором функции $\beta_{1}$ и $\beta_{2}$ удовлетворяют условиям теоремы 9. Тогда функциональное уравнение типа Коши на кривой Г не имеет решений, отличных от $F(z)=\lambda z$, nрu $|z| \leqslant 1$.

Таким образом, для определения функции $F(z)$ на отрезке $-1 \leqslant z \leqslant 1$ нет необходимости в том, чтобы соотношение Коши (16) выполнялось для всех точек $(x, y)$ из квадрата $K=\{(x, y):|x \pm y| \leqslant 1\}$. Достаточно, как видно из следствия 10 , чтобы равенство $F(x+y)=F(x)+F(y)$ имело место для всех точек какой-либо кривой $Г$ описанного вида. Роль этой кривой может выполнять, например, сторона квадрата $K$

$$
x=(t-1) / 2, \quad y=(t+1) / 2, \quad-1 \leqslant t \leqslant 1 .^{1)}
$$

\section{§6. Об интегральном уравнении, связанном с одной геометрической задачей}

Задачи восстановления функции в заданной области $D$ по значениям ее интегралов по какому-либо семейству $\left\{D_{q}\right\}$ подмножеств в $D$ всегда вызывают интерес не только как объект чистого анализа, но и многими приложениями в практических дисциплинах. Наиболее яркий пример подобной связи - задача $\mathrm{Pa}-$ дона и томография. Мы покажем в этом параграфе, что с помощью полученных выше результатов одна из таких задач может быть решена в случае ограниченной области с кусочно-гладкой границей.

Мы начнем с постановки общей задачи. Пусть $\ell_{1}$ и $\ell_{2}-$ неособые гладкие трансверсальные векторные поля в диске $S \in \mathbb{R}^{2}$. Рассмотрим криволинейный треугольник $D=O A_{1} A_{2}$, у которого стороны $O A_{1}$ и $O A_{2}$ совпадают с траекториями векторных полей $\ell_{1}$ и $\ell_{2}$ соответственно, а сторона $A_{1} A_{2}$ представляет собой произвольную неособую гладкую кривую $\Gamma$, трансверсальную обоим полям в точках $A_{1}$ и $A_{2}$. В дополнение к этому предполагается, что замыкание $\bar{D}$ области $D$ удовлетворяет следующим условиям:

1) для любой точки $p \in \bar{D}$ траектория поля $\ell_{j}$, проходящая через $p$, пересекает $O A_{k}$ в точке $\pi_{k} p, j \neq k, 1 \leqslant j, k \leqslant 2$;

2 ) множество $\bar{D}$ является $\ell_{j}$-выпуклым, $j=1,2$; это значит, что если точки $p$ и $q$ из $\bar{D}$ лежат на какой-либо траектории $\gamma_{j}$ поля $\ell_{j}$, то все точки $r \in \gamma_{j}$, расположенные между $p$ и $q$, также принадлежат $\bar{D}$.

Для любой точки $q \in \Gamma$ обозначим через $D_{q}$ криволинейный параллелограмм $q q_{1} O q_{2}$, где $q_{j}=\pi_{j} q, j=1,2$. Условия 1) и 2) гарантируют включение $\bar{D}_{q} \subset D$ при всех $q \in \Gamma$. Упоминавшаяся выше геометрическая задача о восстановлении функции в $D$ принимает в рассматриваемом здесь случае форму интегрального уравнения

$$
\text { Af }:=\int_{D_{q}} f d \sigma=h(q), \quad q \in \Gamma .
$$

1) Когда эта работа была уже подготовлена к печати, автору стало известно, что результат следствия 10 при совершенно иных предположениях относительно функции $\beta_{j}$ был ранее получен другим методом в работе [5]. 
Здесь $\sigma$ обозначает какую-либо меру на $S$, а $h \in C(\Gamma), f \in C(\bar{D})$ - заданная и искомая функции. Общая проблема, сформулированная в книге [1], выглядит следующим образом применительно к этому уравнению: для каких пространств функций $f$ и $h$ отображение $A: f \mapsto h$ является взаимно однозначным и какие функции $h$ представляются интегралом (27). Что касается ответа на второй вопрос, то, как следует из (27), любая такая функция $h$ принадлежит пространству $\mathscr{H}(\Gamma)=\left(C^{2} \cap C_{0}\right)(\Gamma)$ всех дважды дифференцируемых функций, равных нулю на границе $\partial Г$. Поэтому наилучший возможный ответ на первый вопрос состоит в описании подпространств $\mathscr{L}(D)$ пространства $C(\bar{D})$, для которых отображение $A: \mathscr{L}(D) \rightarrow \mathscr{H}(\Gamma)$ является взаимно однозначным. Такие подпространства, разумеется, могут быть определены многими способами. Здесь будет рассмотрен один класс подпространств, естественно возникающий в упоминавшихся выше краевых задачах для гиперболических уравнений (см. §7).

ОПрЕДЕЛЕНИЕ. Пусть $\ell-$ неособое гладкое векторное поле в $S$. Мы обозначим через $C_{\langle\ell\rangle}(D)$ подмножество тех функций из $C(\bar{D})$, которые сохраняют постоянное значение на каждой траектории поля $\ell$.

Размеры настоящей статьи позволяют рассмотреть только тот случай, когда $\ell=r_{1} \ell_{1}+r_{2} \ell_{2}, r_{1} r_{2}>0$ и коэффициенты $r_{1}, r_{2}$ постоянны. ${ }^{1)}$ Однако в этой ситуации удается получить исчерпывающее решение обсуждаемой задачи: мы приводим необходимое и достаточное условие на кривую $Г$, обеспечивающее однозначность оператора $A: C_{\langle\ell\rangle}(D) \rightarrow \mathscr{H}(\Gamma)$.

Чтобы сформулировать это условие, наряду с проекторами $\pi_{j}, j=1,2$, вдоль полей $\ell_{k}, j \neq k$, рассмотрим проектор $\pi_{\ell}: \bar{D} \rightarrow \Gamma$ вдоль траекторий поля $\ell$. Другими словами, для любой точки $p \in \bar{D}$ ее проекция $\pi_{\ell} p$ находится на пересечении траектории поля $\ell$, проходящей через $p$, и кривой Г. Введем два отображения на $\Gamma$

$$
\zeta_{1}=\pi_{\ell} \circ \pi_{1} \quad \text { и } \quad \zeta_{2}=\pi_{\ell} \circ \pi_{2}
$$

и обозначим через $\Phi_{\zeta}$ некоммутативную полугруппу отображений на $\Gamma$, порождаемую $\zeta_{1}$ и $\zeta_{2}$ и аналогичную полугруппе $\Phi_{\delta}$, рассмотренной в $\S 2$. Как и в случае $\Phi_{\delta}$, мы вводим с помощью отображений $\zeta_{1}$ и $\zeta_{2}$ понятие орбиты в $\Gamma$ как последовательности точек $\left(q_{1}, \ldots, q_{n}, \ldots\right)$, таких, что $q_{k+1}=\zeta_{j_{k}} q_{k}, k=1,2, \ldots$ Как и прежде, определяются направляющие множества

$$
\mathscr{T}_{j}=\left\{q \in \Gamma \mid \ell_{j}(q) \in T_{q}(\Gamma)\right\}, \quad j=1,2,
$$

и $\mathscr{T}=\mathscr{T}_{1} \cup \mathscr{T}_{2}$. Дословно повторяя сказанное в $\S 2$, мы определяем периодические, $\mathscr{T}$-направленные, а также $\mathscr{T}$-правильные орбиты, отвечающие полугруппе $\Phi_{\zeta}$, и вводим обозначение $\mathfrak{N}_{\zeta}$ для множества $\mathscr{T}$-направленных $\mathscr{T}$-правильных циклов рассмотренного вида. Мы предполагаем, по аналогии с условием (4), что если оба множества $\mathscr{T}_{1}$ и $\mathscr{T}_{2}$ бесконечны, то любые точки $\tau_{1} \in \mathscr{T}_{1}^{\prime}$ и $\tau_{2} \in \mathscr{T}_{2}^{\prime}$ расположены на $\Gamma$ в таком порядке: $A_{1}, \tau_{1}, \tau_{2}, A_{2}$. Основной результат настоящего параграфа составляет следующая

ТЕОрема 11. При описанных ограничениях на область $D$ и на векторнье поля $\ell_{1}, \ell_{2}$ и $\ell$ для любой функции $h \in \mathscr{H}(\Gamma)$ сущзествует единственное решение $f \in C_{\langle\ell\rangle}(D)$ уравнения (27) в том и только в том случае, когда

\footnotetext{
1) Случай переменных $r_{1}, r_{2}$ изучается в [3].
} 
$\mathfrak{N}_{\zeta}=\varnothing$. Обратный оператор $A^{-1}: h \mapsto f$ является непрерывным оператором из $\mathscr{H}(\Gamma)$ в $C_{\langle\ell\rangle}(D)$.

ДокАЗАТЕЛЬСтво. Не ограничивая общности, можно предположить, что векторные поля $\ell_{1}$ и $\ell_{2}$ параллельны координатным осям $x_{1}$ и $x_{2}$ и в качестве $D$ рассмотреть криволинейный треугольник, две стороны которого совпадают с отрезками $\left\{x_{1} \mid 0 \leqslant x_{1} \leqslant 1\right\}$ и $\left\{x_{2} \mid 0 \leqslant x_{2} \leqslant 1\right\}$ осей $x_{1}$ и $x_{2}$. Что касается третьей стороны $\Gamma$, то предполагается, что это гладкая неособая кривая, трансверсальная осям $x_{1}$ и $x_{2}$. Рассмотрим произвольное параметрическое представление этой кривой $x_{1}=\alpha_{1}(z), x_{2}=\alpha_{2}(z), z \in I$, и введем обозначение $\alpha(z)=\left(\alpha_{1}(z), \alpha_{2}(z)\right)$. Можно показать, что в силу топологических ограничений $1)$ и 2$)$ на область $D$ функции $\alpha_{j}(z)$ удовлетворяют условиям

$$
\alpha_{1}^{\prime}(z) \geqslant 0, \quad \alpha_{2}^{\prime}(z) \leqslant 0 \quad \text { и } \quad\left|\alpha_{1}^{\prime}(z)\right|+\left|\alpha_{2}^{\prime}(z)\right|>0, \quad z \in I,
$$

при том, что $\alpha(1)=(1,0)$. Введем также функцию $\omega(x)=r_{2} x_{1}-r_{1} x_{2}$ в $\bar{D}$ и обозначим через $\omega_{\Gamma}$ ее ограничение на $Г$. Роль функции $\omega$ становится ясной, если заметить, что она постоянна на траекториях поля $\ell$. Поэтому любая функция $g \in C_{\langle\ell\rangle}(D)$ представима в виде $g=f \circ \omega$, где $f-$ некоторая функция, непрерывная на отрезке $I_{t}=\left[-r_{1}, r_{2}\right]$. Нам понадобятся в дальнейшем также функции $\omega_{1}(x)=\omega\left(x_{1}, 0\right)$ и $\omega_{2}(x)=\omega\left(0, x_{2}\right)$. Заметим, что функция $\sigma=\omega_{\Gamma} \circ \alpha: I \rightarrow I_{t}$ обратима, так как $\sigma^{\prime}(z)>0$ в силу неравенств (28). В координатной форме уравнение (27) записывается следующим образом:

$$
\int_{0}^{\alpha_{1}(z)} \int_{0}^{\alpha_{2}(z)} f(\omega(x)) d x_{2} d x_{1}=h(z), \quad z \in I .
$$

Введем новую неизвестную функцию $F(t)=-\int_{0}^{t} f(s)(t-s) d s / r_{1} r_{2}$. Подставляя в (29) $F$ вместо $f$, мы после рутинных вычислений приходим к следующему функциональному уравнению для неизвестной функции $F \in C\left(I_{t}\right)$ :

$$
F(\omega \circ \alpha)-F\left(\omega_{1} \circ \alpha\right)-F\left(\omega_{2} \circ \alpha\right)=h .
$$

Поскольку $F^{\prime \prime}(t)=-f(t) / r_{1} r_{2}$ и $F(0)=F^{\prime}(0)=0$, отображение $f \mapsto F$ взаимно однозначно. Теорема, следовательно, будет доказана, если мы установим однозначную разрешимость уравнения (30). Введем новые отображения

$$
\rho_{1}=\omega_{1} \circ \alpha \circ \sigma^{-1} \quad \text { и } \rho_{2}=-\omega_{2} \circ \alpha \circ \sigma^{-1}
$$

отрезка $I_{t}$ в себя, с помощью которых уравнение (30) переписывается в виде

$$
F(t)-F\left(\rho_{1}(t)\right)-F\left(\rho_{2}(t)\right)=h\left(\sigma^{-1}(t)\right), \quad t \in I_{t} .
$$

Замечательно, что функции $\rho_{1}$ и $\rho_{2}$, инвариантным образом связанные с геометрической задачей (27), обладают всеми свойствами функций $\beta_{1}$ и $\beta_{2}$, постулированными в предыдущем параграфе. При этом, поскольку $\rho_{1}(t)+\rho_{2}(t)=t$ при всех $t \in I_{t}$, уравнение (31) совпадает с уравнением (17), а значит, представляет собой функциональное уравнение типа Коши на отрезке $I_{t}$. Обозначим через $\Phi_{\rho}$ полугруппу отображений в $I_{t}$, порожденную образующими $\rho_{1}$ и $\rho_{2}$, и через $O_{\rho}$ соответствующее множество орбит. Введем в рассмотрение направляющие множества $\mathscr{T}_{\rho_{j}}=\left\{t \in I_{t} \mid \rho_{j}^{\prime}(t)=0\right\}, j=1,2$, и $\mathscr{T}_{\rho}=\mathscr{T}_{\rho_{1}} \cup \mathscr{T}_{\rho_{2}}$. Мы можем теперь стандартным способом определить $\mathscr{T}_{\rho}$-направленные, периодические и $\mathscr{T}_{\rho}$-правильные орбиты и ввести обозначения $\mathfrak{N}_{\rho}^{\mathscr{T}_{\rho}}$ для совокупности всех орбит из $O_{\rho}$, являющихся одновременно $\mathscr{T}_{\rho}$-направленными, периодическими и 
$\mathscr{T}_{\rho}$-правильными. В силу основного свойства функции $\omega-$ постоянства ее значений на траекториях поля $\ell-$ мы имеем $\omega_{j} \circ \alpha=\omega_{\Gamma} \circ \zeta_{j} \circ \alpha$ при $j=1,2$. Подставляя эти равенства в формулы, определяющие $\rho_{1}$ и $\rho_{2}$, мы получим, что $\rho_{j}=\omega_{\Gamma} \circ \zeta_{j} \circ \omega_{\Gamma}^{-1}$ при тех же $j$ и, следовательно, $\rho_{J}=\omega_{\Gamma} \circ \zeta_{J} \circ \omega_{\Gamma}^{-1}$ для любого мультииндекса $J$. Отсюда немедленно следует, что если $\mathscr{O}=\left(q_{1}, \ldots, q_{n+1}\right)$

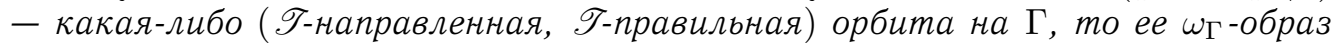
представляет собой ( $\mathscr{T}_{\rho}$-направленную, $\mathscr{T}_{\rho}$-правильную) орбиту на $I_{t}$. Это позволяет заключить, что множества $\mathfrak{N}_{\zeta}$ и $\mathfrak{N}_{\rho}^{\mathscr{T}_{\rho}}$ могут быть пустыми только одновременно. Но тогда из условия теоремы 11 следует, что $\mathfrak{N}_{\rho}^{\mathscr{T}_{\rho}}=\varnothing$, и для завершения ее доказательства в части разрешимости остается воспользоваться результатом теоремы 9. Чтобы доказать непрерывность оператора $A^{-1}$, достаточно воспользоваться непрерывностью оператора $h\left(\sigma^{-1}(t)\right) \mapsto F(t)$ в пространстве $C^{2}(I)$, определенного уравнением $(31)$, и определением функции $F$.

Остается проверить необходимость условия $\mathfrak{N}_{\zeta}=\varnothing$. Если $\mathfrak{N}_{\zeta} \neq \varnothing$, то $\mathfrak{N}_{\rho}^{\mathscr{T}_{\rho}} \neq \varnothing$, и, значит, существует $\mathscr{T}_{\rho}$-правильная периодическая орбита $\mathscr{O}=\left(t_{1}, \ldots, t_{n+1}\right)$, целиком расположенная в критическом множестве $\mathscr{T}_{\rho}$. Заменим в $(31) h\left(\sigma^{-1}(t)\right)$ функцией $H(t)$ и продифференцируем полученное равенство. Полагая $F^{\prime}=G$, приходим к соотношению

$$
G(t)-\rho_{1}^{\prime}(t) G\left(\rho_{1}(t)\right)-\rho_{2}^{\prime}(t) G\left(\rho_{2}(t)\right)=H^{\prime}(t) .
$$

Заметим теперь, что, согласно выбору точек $t_{j}, j=1, \ldots, n+1$, мы имеем при всех значениях $k=1, \ldots, n$

$$
t_{n+1}=t_{1} \quad \text { и } \quad t_{k+1}=\rho_{j_{k}}\left(t_{k}\right), \quad \rho_{j_{k}^{\prime}}^{\prime}\left(t_{k}\right)=0 \quad \text { для } j_{k}^{\prime} \neq j_{k} .
$$

Подставим в уравнение $(32) t_{1}$ вместо $t$. Тогда одно из чисел $\rho_{j}^{\prime}\left(t_{1}\right)$ равно нулю, в то время как другое число $\rho_{j^{\prime}}^{\prime}\left(t_{1}\right)$ равно единице. При этом из определения $\mathscr{T}$-правильности следует, что $t_{2}=\rho_{j_{k}^{\prime}}\left(t_{1}\right)$. В результате получаем $G\left(t_{1}\right)-G\left(t_{2}\right)=$ $H^{\prime}\left(t_{1}\right)$. Продолжая эту процедуру, мы приходим к цепочке равенств $G\left(t_{2}\right)-$ $G\left(t_{3}\right)=H^{\prime}\left(t_{2}\right), \ldots, G\left(t_{n}\right)-G\left(t_{1}\right)=H^{\prime}\left(t_{n}\right)$, где на последнем шаге использована периодичность орбиты $\mathscr{O}$. Складывая эти равенства, мы получаем

$$
\sum_{j=1}^{n} H^{\prime}\left(t_{j}\right)=0 \text {. }
$$

Таким образом, полученное соотношение является необходимым условием разрешимости уравнения (27) при наличии $\mathscr{T}$-правильного $\mathscr{T - н а п р а в л е н н о г о ~ ц и к л а . ~}$ Тем самым необходимость условия $\mathfrak{N}_{\zeta}=\varnothing$ доказана, что и завершает доказательство теоремы 11.

ЗАмЕЧАНИЕ. Полученный результат позволяет интерпретировать множество орбит $\mathfrak{N}_{\zeta}$ как препятствие к построению решения уравнения $(27)$.

\section{§7. О разрешимости некоторых граничных задач для гиперболических дифференциальных уравнений с данными на всей границе}

В качестве еще одного приложения результатов, относящихся к уравнению (1), рассмотрим в $(x, y)$-плоскости произвольный однородный $x$-строго гиперболический оператор $P\left(\partial_{x}, \partial_{y}\right)$ третьего порядка с постоянными коэффициентами. Такой оператор единственным образом представляется в виде $P\left(\partial_{x}, \partial_{y}\right)=$ 
$a\left(\partial_{x}-a_{1} \partial_{y}\right)\left(\partial_{x}-a_{2} \partial_{y}\right)\left(\partial_{x}-a_{3} \partial_{y}\right)$, где $a, a_{1}, a_{2}$ и $a_{3}$ суть вещественные числа и $a_{j} \neq a_{k}$, если $j \neq k$. Характеристиками оператора $P$ являются прямые линии $y+a_{1} x=$ const, $y+a_{2} x=$ const, $y+a_{3} x=$ const. Обозначим через $\ell_{1}, \ell_{2}$ и $\ell_{3}$ соответственно векторные поля в $\mathbb{R}^{2}$, параллельные этим прямым. Пусть $O A_{1}, O A_{2}$ и $O A_{3}$ - произвольная тройка соседних характеристических лучей, выходящих из некоторой точки $O$ (таких лучей имеется 6 ), причем луч $O A_{3}$ расположен между $O A_{1}$ и $O A_{2}$.

Рассмотрим криволинейный треугольник $D=O A_{1} A_{2}$ со сторонами $O A_{1}, O A_{2}$ и $\Gamma=A_{1} A_{2}$, в котором $\Gamma$ представляет собой гладкую кривую без особенностей, трансверсальную $O A_{1}$ и $O A_{2}$. Предполагается, что замыкание $\bar{D}$ удовлетворяет условиям 1) и 2) из $\$ 6$. Рассмотрим следующую краевую задачу: по заданным функциялм $F \in C(\bar{D})$ и $h \in C(\partial D)$ найти функциню $и$, такую, что

$$
P\left(\partial_{x}, \partial_{y}\right) u=F \quad \text { в } D, \quad u=h \quad \text { на } \partial D .
$$

Чтобы сформулировать основной результат, рассмотрим полугруппу $\Phi_{\zeta}$ отображений в $\Gamma$, введенную в $\$ 6$, с $\ell=\ell_{3}$. Направляющие множества $\mathscr{T}_{1}$ и $\mathscr{T}_{2}$ оказываются в новой ситуации не чем иным, как характеристическими подмножествами в Г. Введем множество $\mathfrak{N}_{\zeta}$ всех $\mathscr{T}_{\zeta}$-правильных периодических орбит полугруппы $\Phi_{\zeta}$, состоящих из характеристических точек. Обозначим через $C^{k}(\partial D)$ совокупность непрерывных на $\partial D$ функций, ограничения которых на все стороны треугольника $O A_{1} A_{2} k$ раз непрерывно дифференцируемы, $k>2$.

Теорема 12 (ср. [2]). Допустим, что множества $\mathscr{T}_{1}$ и $\mathscr{T}_{2}$ удовлетворяют условию, сформулированному перед теоремой 11. Тогда для любых функций $F \in C^{k}(\bar{D}), \quad h \in C^{k+2}(\partial D), k \geqslant 1$, существует единственное решение $u \in$ $C^{k+2}(\bar{D})$ задачи (34) в том и только том случае, когда $\mathfrak{N}_{\zeta}=\varnothing$. Обратный оператор $(F, h) \mapsto$ ограничен в соответствующей паре пространств.

ДокАЗАТЕЛьство. Ограничимся случаем $F=0$. Очевидно, что линейная замена переменных сводит (34) к задаче

$$
\left(r_{1} \partial_{x}+r_{2} \partial_{y}\right) \partial_{x} \partial_{y} u=0 \quad \text { в } D, \quad u=h \quad \text { на } \partial D
$$

с $r_{1} r_{2}>0$. Граница области $D$ теперь состоит из трех участков:

$$
\begin{gathered}
\Gamma_{1}=\{(x, y) \mid y=0,0 \leqslant x \leqslant 1\}, \quad \Gamma_{2}=\{(x, y) \mid x=0,0 \leqslant y \leqslant 1\} \quad \text { и } \\
\Gamma=\left\{(x, y) \mid x=\alpha_{1}(t), y=\alpha_{2}(t),-1 \leqslant t \leqslant 1\right\},
\end{gathered}
$$

причем $\alpha_{1}(-1)=0, \alpha_{1}(1)=1, \alpha_{2}(-1)=1, \alpha_{2}(1)=0$. Пусть $h=h_{1}(x)$ на $\Gamma_{1}$, $h=h_{2}(y)$ на $\Gamma_{2}$ и $h=h_{3}(x, y)$ на $\Gamma$. В силу непрерывности функции $h$ на $\partial D$ выполняются условия совместимости

$$
h_{1}(0)=h_{2}(0), \quad h_{1}(1)=h_{3}(1,0), \quad h_{2}(1)=h_{3}(0,1),
$$

Используя постулированные свойства области $D$, легко проверить, что функция

$$
u(x, y)=\int_{0}^{x}\left(\int_{0}^{y} F\left(r_{2} s-r_{1} t\right) d t\right) d s+h_{1}(x)+h_{2}(y)-h_{1}(0), \quad 0 \leqslant x, y \leqslant 1,
$$

удовлетворяет уравнению в $(35)$ и условию $u=h$ на части границы $\Gamma_{1} \cup \Gamma_{2}$ при любой функции $F \in C^{1}\left(\left[-r_{2}, r_{1}\right]\right)$. Поскольку необходимо, чтобы выполнялось 
условие $u=h_{3}$ на $\Gamma$, мы приходим теперь к интегральному уравнению

$$
\int_{0}^{\alpha_{1}(t)}\left(\int_{0}^{\alpha_{2}(t)} F\left(r_{2} x-r_{1} y\right) d y\right) d x=H(t), \quad-1 \leqslant t \leqslant 1
$$

в котором, как и в $\$ 6$, равенства $x=\alpha_{1}(t), y=\alpha_{2}(t), t \in I$, служат параметрическим представлением кривой $\Gamma$, а $H(t)=-h_{1}\left(\alpha_{1}(t)\right)-h_{2}\left(\alpha_{2}(t)\right)+$ $h_{3}\left(\alpha_{1}(t), \alpha_{2}(t)\right)+h_{1}(0)$. Важно отметить, что функция $H$, порожденная произвольной непрерывной $k$ раз кусочно-дифференцируемой функцией $h$, принадлежит пространству $\mathscr{H}(I)=\left(C^{2} \cap C_{0}\right)(I)$. Это следует из (36). Верно и обратное: функция $u$, определенная формулой (37), является решением задачи (35). Таким образом, эта последняя оказывается эквивалентной интегральному уравнению (38), которое есть не что иное, как уравнение (29). Однозначная разрешимость задачи (35) для произвольных $h$ нужного вида при выполнении условия $\mathfrak{N}_{\zeta}=\varnothing$ непосредственно следует из теоремы 11.

Чтобы проиллюстрировать полученный результат, рассмотрим задачу (35) в областях $D_{1}, D_{2}$ и $D_{3}$ следующего вида:
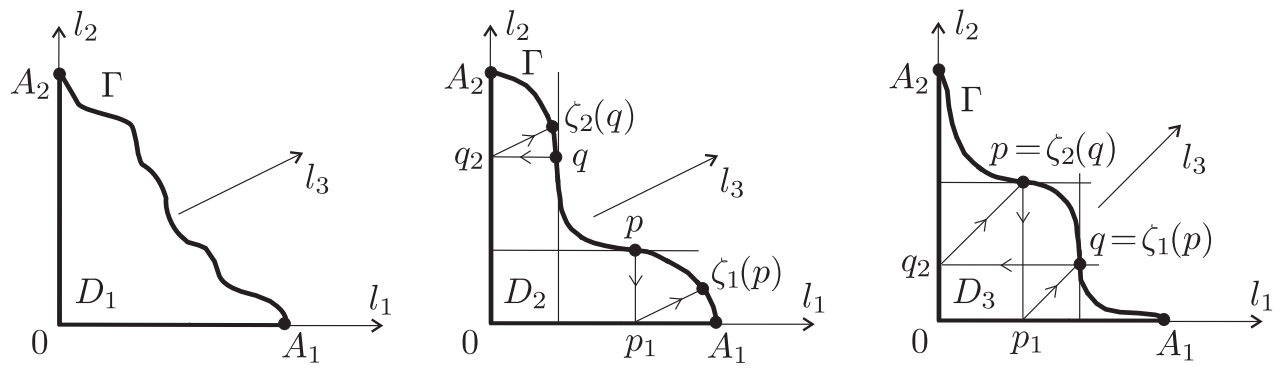

На этих рисунках только точки $p$ и $q$ являются характеристическими. Легко проверяется, что $\mathfrak{N}_{\zeta}=\varnothing$ для областей $D_{1}$ и $D_{2}$, и, значит, задача (35) оказывается корректно поставленной в $D_{1}$ и $D_{2}$. С другой стороны, в случае области $D_{3}$ множество $\mathfrak{N}_{\zeta}$ содержит (единственную) характеристическую $\mathscr{T}$-правильную периодическую траекторию $(p, q, \rho)$, что делает теорему 12 неприменимой.

В заключение проверим, что по крайней мере в случае конечного характеристического множества $\mathscr{T}=\mathscr{T}_{1} \cup \mathscr{T}_{2}$ условие $\mathfrak{N}_{\zeta}=\varnothing$ корректной разрешимости задачи (34) является типическим. Это означает, что в пространстве $C^{1}$-кривых Г вида

$$
\Gamma=\left\{(x, y) \mid x=\delta_{1}(t), y=\delta_{2}(t), t \in I\right\},
$$

где функции $\delta_{1}$ и $\delta_{2}$ описаны в $\$ 2$, те кривые, которые удовлетворяют этому условию, образуют множество второй категории в некотором полном метрическом пространстве.

Если одно из множеств $\mathscr{T}_{1}, \mathscr{T}_{2}$ пусто, то типичность условия $\mathfrak{N}_{\zeta}=\varnothing$ очевидна, как очевидна типичность условия нехарактеристичности. Рассмотрим простейший случай одноточечных множеств $\mathscr{T}_{1}=\{p\}$ и $\mathscr{T}_{2}=\{q\}$ (см. выше области $D_{2}$ и $\left.D_{3}\right)$. По определению множество $\mathfrak{N}_{\zeta}$ может состоять в этой ситуации не 
более чем из одной орбиты $\mathscr{O}=(p, q, p)$ (орбита $(q, p, q)$ естественно отождествляется с $\mathscr{O})$, Поэтому рассматриваемая задача может быть теперь переформулирована следующим образом. ${ }^{1)}$ Пусть $C^{1}=C^{1}(I) \times C^{1}(I)$, и пусть

$$
B=\left\{\left(\delta_{1}, \delta_{2}\right) \in C^{1} \mid \delta_{1}^{\prime} \geqslant 0, \delta_{2}^{\prime} \geqslant 0, \delta_{1}^{\prime}(s)=\delta_{2}^{\prime}(t)=0\right\},
$$

где множества соответствующих значений $s$ и $t$ могут меняться от одной пары $\left(\delta_{1}, \delta_{2}\right)$ к другой. Очевидно, что $B$ замкнуто в $C^{1}$, а потому $B$ является полным метрическим пространством. Пусть $W \subset B$ состоит из таких функций $\left(\delta_{1}, \delta_{2}\right)$, что для каждой из них имеется единственная пара $(a, b) \in I \times I$ с $\delta_{1}^{\prime}(a)=\delta_{2}^{\prime}(b)=0$. Наконец, определим множество $V \subset W$ таких пар $\left(\delta_{1}, \delta_{2}\right)$, что $\delta_{2}(a)=b$ и $\delta_{1}(b)=a$. Легко проверяется, что $W$ плотно в $B$ и что $V$ является множеством первой категории в $B$. Покажем, что $W \backslash V-$ множество второй категории в $B$ (что и решает исходную задачу). Достаточно проверить, что $W$ является множеством типа $G_{\delta}$. Это следует из представления $B \backslash W=\bigcup_{n=1}^{\infty} A_{n}$, в котором каждое множество

$A_{n}=\left\{\left(\delta_{1}, \delta_{2}\right) \in B \mid \operatorname{diam}\left\{t \in I \mid \delta_{1}^{\prime}(t)=0\right\} \geqslant \frac{1}{n}\right.$ или $\left.\operatorname{diam}\left\{t \in I \mid \delta_{2}^{\prime}(t)=0\right\} \geqslant \frac{1}{n}\right\}$ очевидно замкнуто, поскольку $W=\bigcap_{n=1}^{\infty}\left(B \backslash A_{n}\right)$.

\section{ЛИТЕРАТУРА}

1. Gelfand I., Graev M., Vilenkin N. Integral geometry and representation theory. New York, Academic Press, 1966.

2. Paneah $B$. On a problem in integral geometry connected to the Dirichlet problem for hyperbolic equations, Intern. Math. Res. Notices, No. 5, 213-222 (1997).

3. Paneah B. On a New Problem in Integral Geometry Related to Boundary Problems for Hyperbolic Differential Equations. Preprint 2001/25, Institut für Matematik, Uni Potsdam, 2001.

4. Paneah B. Dynamical methods in the general theory of Cauchy type functional equations. Israel. Math. Conf. Proc., Vol. 17, 2003.

5. Zdun M. On the uniqueness of solutions of $\varphi(x+f(x))=\varphi(x)+\varphi(f(x))$. Aequationes Math., 8, 229-232 (1972).

Department of Mathematics, Technion

Haifa, Israel

Поступило в редакцию

e-mail: peter@techunix.technion.ac.il

27 января 2002 г.

1) Последующим рассуждением я обязан проф. Й. Беньямини. 